Témoigner Témoigner. Entre histoire et mémoire

Getuigen Revue pluridisciplinaire de la Fondation Auschwitz

$126 \mid 2018$

Questions sur l'avenir du travail de mémoire

\title{
Dunkirk de Christopher Nolan : le miracle d'une évacuation
}

\section{Brecht Capiau}

Traducteur : Emilie Syssau

\section{OpenEdition \\ Journals}

\section{Édition électronique}

URL : https://journals.openedition.org/temoigner/6955

DOI : $10.4000 /$ temoigner.6955

ISSN : 2506-6390

Éditeur:

Éditions du Centre d'études et de documentation Mémoire d'Auschwitz, Éditions Kimé

Édition imprimée

Date de publication : 2 avril 2018

Pagination : 19-22

ISBN : 978-2-930953-06-9

ISSN : 2031-4183

\section{Référence électronique}

Brecht Capiau, «Dunkirk de Christopher Nolan : le miracle d'une évacuation », Témoigner. Entre histoire et mémoire [En ligne], 126 | 2018, mis en ligne le 24 janvier 2022, consulté le 04 février 2022. URL : http://journals.openedition.org/temoigner/6955 ; DOI : https://doi.org/10.4000/temoigner.6955 


\section{DUNKIRK LE MIRACLE D'UNE ÉVACUATION (CHRISTOPHER NOLAN)}

\section{"Wars are not won by evacuations"}

CINÉMA Fin mai 1940. La Drôle de guerre en France touche à son terme. Partout, les troupes britanniques, françaises et belges sont contraintes à la défensive et repoussées en direction de la mer. La British Expeditionary Force (BEF, corps expéditionnaire britannique) et un reliquat de l'armée française, talonnés par les troupes allemandes, se regroupent à Dunkerque en vue d'une évacuation.

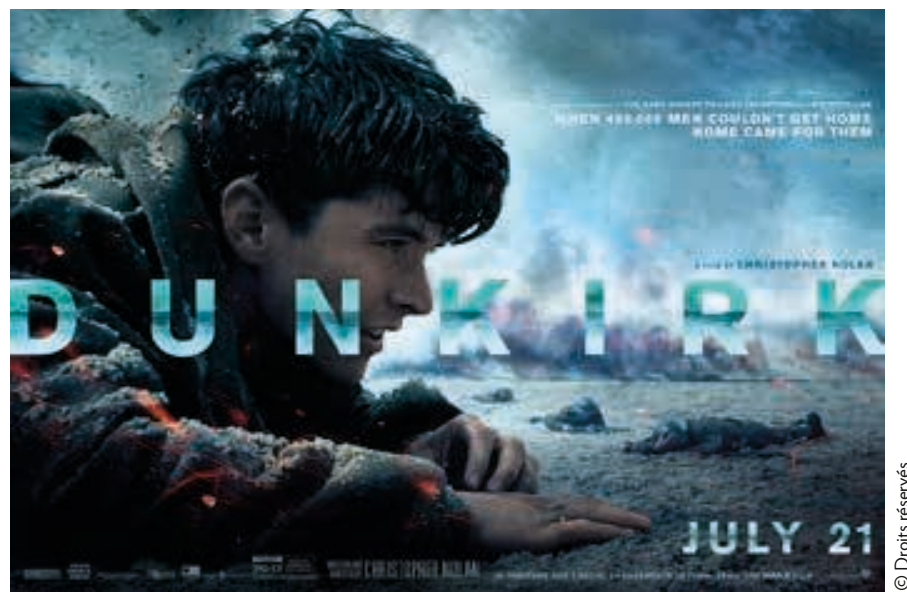

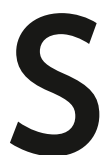
oudain, l'impensable se produit. Le 24 mai, sur les conseils des Feldmarschälle Gerd von Rundstedt et Günther von Kluge, Adolf Hitler ordonne à l'armée allemande de stopper son avancée. Mais cet « ordre d'arrêt des combats » historiquement célèbre offre aux Alliés un laps de temps suffisant pour dresser une ligne de défense autour de Dunkerque et organiser l'opération Dynamo, leur évacuation vers l'Angleterre. Entre le 26 mai et le 4 juin, plus de 330000 soldats, sur un total estimé à 400000 , ont été sauvés de ce qui aurait pu être la plus grande catastrophe militaire de l'histoire mondiale.

(1) «Les guerres ne sont pas gagnées par des évacuations » - Winston Churchill, Premier ministre britannique, discours devant la House of Commons, à propos de l'évacuation de Dunkerque en mai 1940.

\section{LE FILM DE GUERRE REVISITÉ}

L'évacuation de Dunkerque dans les premiers jours de la Seconde Guerre mondiale, tel est le sujet de Dunkirk, le nouveaufilm du réalisateurbritannico-américain Christopher Nolan. L'idée a germé lors d'une traversée de la Manche vers Dunkerque qu'il a effectuée en 1992. Se sentant trop jeune et n'ayant encore aucune expérience des grosses productions cinématographiques, Nolan a alors décidé d'attendre et d'engranger d'abord l'expertise nécessaire à un tel projet. Le produit fini est une véritable surprise sur bien des plans, et montre un réalisateur au sommet de son pouvoir créateur. Pour commencer, Nolan utilise trois perspectives narratives différentes, en trois lieux différents, et selon trois échelles temporelles différentes. Ce système nous est brièvement exposé dans le premier quart du film. 
... La première perspective narrative, intitulée The Mole, suit les revers de Tommy, un jeune soldat britannique qui attend son évacuation sur lune des ur une semaine

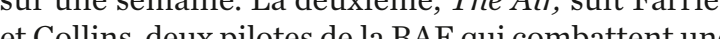
et Collins, deux pilotes de la RAF qui combattent une Luf twaffe. La trisìms sérerschitt Bf 109 de la d'une pa. L'histoire de Mr. Daw d'une part histoire de Mr. Dawson et de son fils Peter, à bord d'un des innombrables Litle Ships of Dunkirk,

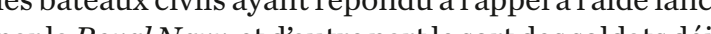
par la Royal Nany, etd autre partle sort des soldats déja do véritablemt un triptyque donnt unstitue donc veritablemét un affronter sur terre, en mer et dans les airs.

L'ensemble est en outre présenté de façon non linéaire: on se retrouve parmi les noyés d'un destroyer rérise et linstant daprès, on assiste à un combat schmitt allemands.
Dunkirk se distingue des autres blockbusters par la Des dialogues et son assurance absolue de narrer l'histoire de façon tres visuelle. On cont le nom despers lerour personir. cectar Gilm leurs actions nide les expliquer aux autres. Un sper a du spectateur à un ou plusieurs personnages. L'envie ou réussi un réussiou de l'atachent pour un personabe de nyme, ou quasi anent pour un personnage anonyt pro on par Clint Eastwood dans la trilogie Dollars interprete par Clint Eastwood dans la trilogie Dollars du cinéaste film froid, gé, in jeunes personnages jeunes personnages. Lattitude de Mr. Dawson envers un personnage atteint du syndrome de stress posttaine, en routevis lon dit long a cet égard. Le capison bateau, découvre en pleine mer un réá, joué

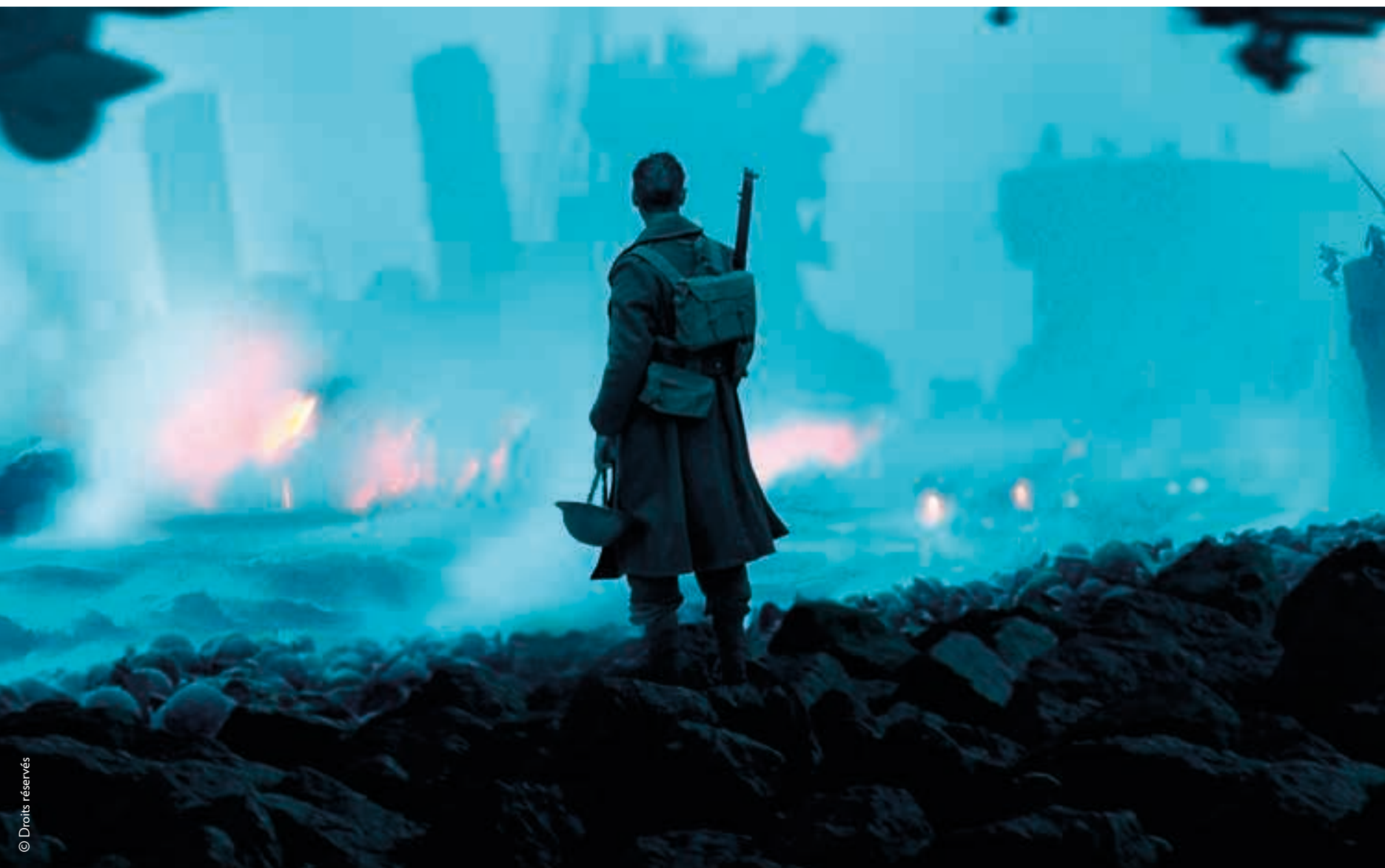

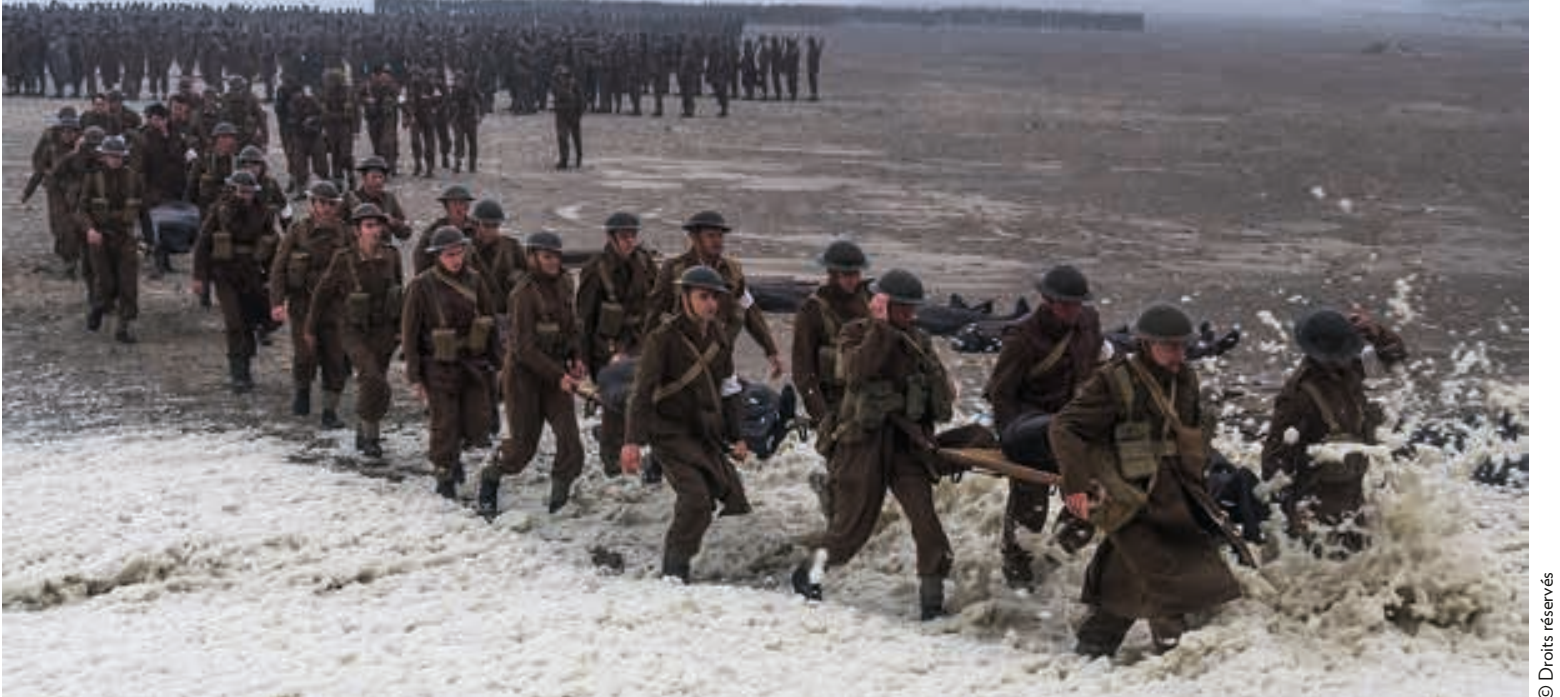

par Cillian Murphy, le regard fixé sur la proue d'un navire en train de sombrer. Il apparaît rapidement navire en train de sombrer. Il apparait rapidement

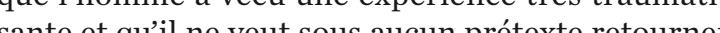
le cu'il ne ver

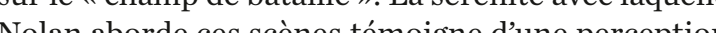
riguë de la psýs hunes témoigne dune perception lo les personnesfragiles que lon voit trop peu aujourd hu ciném toraphique se caractérise splu. Le dispositif IMAX $70 \mathrm{~mm}$, qui, vir

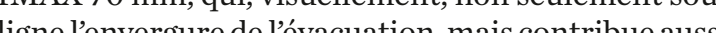
lignelenvergure de lévacuation, mais contribue aus Plus encore qu'un film, Dunhirk est une exirence. expérience.

Dunkirk est également d'une autre trempe que les films de guerre auxquels nous sommes habitués. La pellicule présente notamment plus de similitude avec les films de guerre britanniques des annees 1950 The Cruel Sa (La ters (Les Briseurs de bar ters (Les Briseurs de barrages, de sentimanta d'était d'ailleurs néce absence dix sentimentalisme. Cétait dailleurs nécessaire, car dix ans à peine après la Seconde Guerre mondiale, guerre. Il est donc aussi trìs satisfisant que Nola revienne à cette authenticité d'autrefois. En Europe à septante ans, et le risque existe d'aborder les événements de lépoque à travers des lunettes trop colorées, t plutôt pathétiques. Autre similitude remarquable vec les célèbres films de guerre britanniques : on ne voit pas l'ennemi. Il n'y a aucune image de corps déchquetés par les bonbes ou d'autres atrocités guerrières moins aussi mortellement fatal que la coure estau

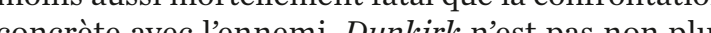
conceste plus une histoire d hommes politiques,

Le célèbre discours «We Shall Fight on The Beaches » (Nous combattrons sur les plages) à la fin du film nest pas prononce par son auteur, Winston lit ceine jo sorte de commentaire irenique de politiques qui lutteront sur les plages, mas bien politiques qui lutteront sur les plages, mais bien les dans cette scène, Nolan s'ats dans la presse. Enfin, dans cette scène, Nolan s'amuse dun jeu de mots sur "Tommy »: ce n'est pas un hasard si, outre le prénom britpensonnage, ils'git dusun

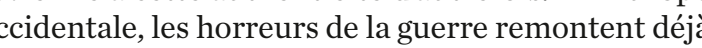
chilaires. Nolan estime ajuste titre que le stress psydu réalie sorte de conmmentaire ironique de la part 


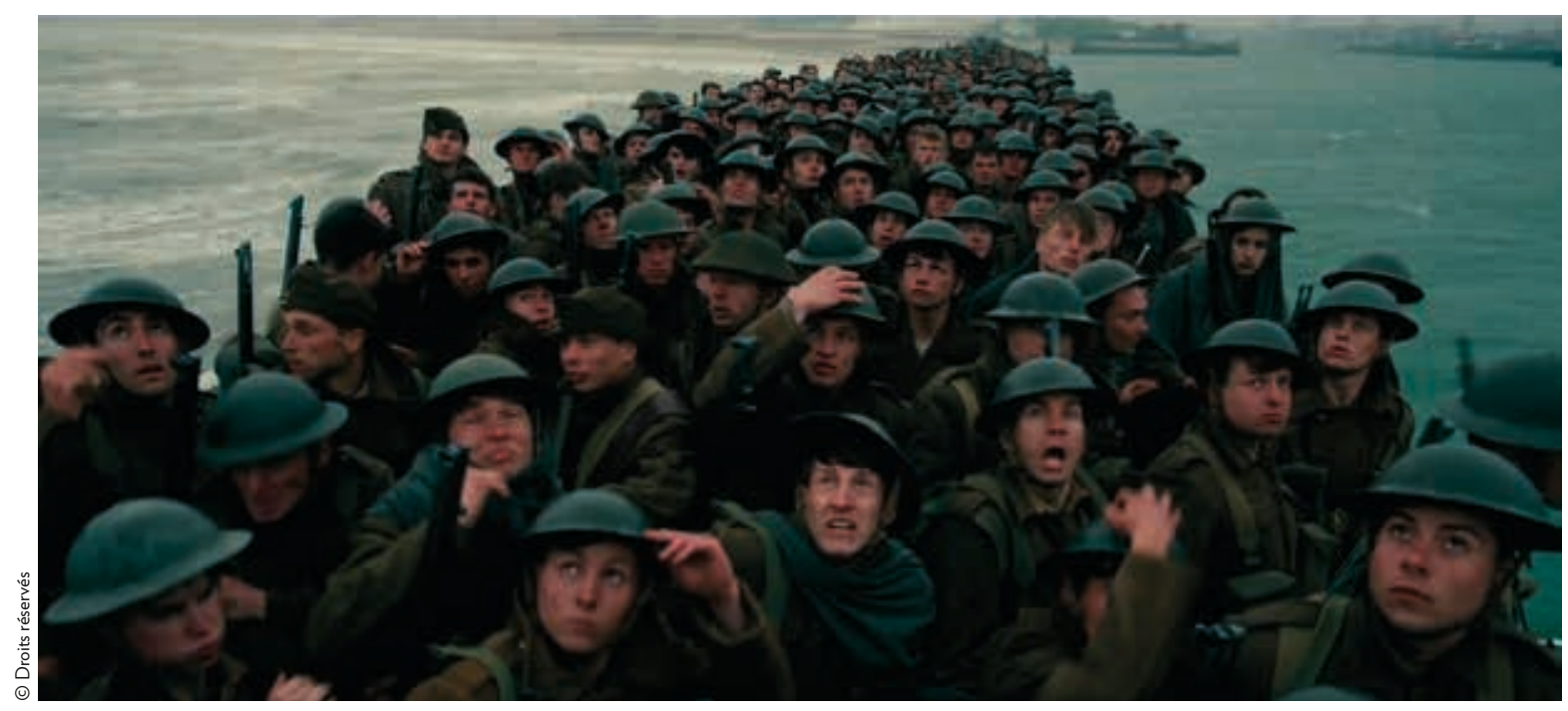

\section{LA PLACE DE DUNKIRK DANS L'CEUVRE DE NOLAN}

"Show them what they have, and then you take it away » (Montrez-leur ce qu'ils ont puis retirez-le-leur), dit le cambrioleur Cobb à son apprenti Bill dans Following (Following, le suiveur, 1998), le premier film de Christopher Nolan. Dès le début de sa carrière, Nolan est intrigué par ce que l'on appelle en anglais des « con artists » : des hommes qui réussissent de façon surprenante à tromper leurs semblables et à échapper aux conséquences de leurs actes. Presque tous les films de Nolan jouent, plus ou moins, avec cette donnée. Parfois littéralement, comme dans The Prestige (Le Prestige, 2006) où deux prestidigitateurs rivaux se disputent autour d'un tour de magie « The Real Transported Man », ou de façon figurée et symbolique comme dans Inception (2010). Dans ce film, Dom Cobb participe à l'espionnage industriel en volant les idées dans l'inconscient de ses « clients ». Dunkirk s'inscrit merveilleusement dans cette lignée. Pour Nolan, il s'agit en effet d'un authentique tour de magie. Au début du film, le réalisateur montre ce que les Allemands ont virtuellement entre les mains : 400000 soldats épuisés, affamés, amassés sur une plage. À la fin ne restent sur une plage déserte que quelques dizaines de casques " assiette à soupe » typiquement britanniques. Les Alliés font littéralement « disparaître » les soldats restés, et les Allemands se retrouvent les mains vides.

On peut néanmoins parler d'une variation sur le thème. C'est tout d'abord le film le plus court depuis le début de la carrière de Christopher Nolan. Le scénario original ne comptait que septante-six pages - la moitié d'un scénario moyen. En outre, Nolan délaisse l'emphase de ses précédents films et opte pour une forme épurée afin de relater un récit basé sur des faits authentiques. Un récit qui n'est jamais démonstratif et qui, par son commentaire historique sommaire, donne au public la possibilité d'entreprendre ses propres recherches. Un récit qui, malgré le traitement restreint de ses personnages, est plein d'émotion en raison du caractère extraordinaire de la situation esquissée. Toute information ou dialogue supplémentaires rendraient rapidement l'ensemble pathétique ou sentimental. Enfin, le film Dunkirk n'a pas été réalisé dans le cadre d'une tantième commémoration de l'évacuation de Dunkerque. Le projet n'a donc pas été trop contaminé par des suggestions politiques ni par des sentiments patriotiques. Certes, monsieur Churchill, on ne peut remporter des guerres par des évacuations. Elles permettent cependant d'assurer l'avenir. I

Brecht Capiau

Traduit du néerlandais par Emilie Syssau

\section{Plus d'infos}

Dunkirk de Christopher Nolan (2017)

Avec Fionn Whitehead (Tommy), Tom Hardy (Farrier), Mark Rylance (Mr. Dawson), etc. - 105 minutes. 